\title{
The 1/N Expansion of Colored Tensor Models
}

\author{
Razvan Gurau
}

\begin{abstract}
In this paper, we perform the $1 / N$ expansion of the colored three-dimensional Boulatov tensor model. As in matrix models, we obtain a systematic topological expansion, with increasingly complicated topologies suppressed by higher and higher powers of $N$. We compute the first orders of the expansion and prove that only graphs corresponding to three spheres $S^{3}$ contribute to the leading order in the large $N$ limit.
\end{abstract}

\section{Introduction}

Random matrix models are a very important tool in modern theoretical physics. They arise in the most diverse contexts, from the seminal work of 't Hooft [1] on confinement, to string theory [2,3], two-dimensional gravity [4], critical phenomena [5-7], black hole physics [8], and the list goes on. The crucial feature of matrix models is their $1 / N$ topological expansion [9]. It is only due to this expansion that notions like "planar diagrams", "double scaling limit", etc. are meaningful. Most importantly, the simplest topologies (planar graphs dual to spheres $S^{2}$ ) dominate this expansion. For all their interest, random matrix models are only two-dimensional. To approach a more realistic physical setting, one generalizes them in higher dimensions to random tensor models [10-12] and Group Field Theories (GFT) [13,14].

In three dimensions, the Feynman graphs of GFT are built from vertices dual to tetrahedra and propagators encoding the gluing of tetrahedra along boundary triangles. Parallel to ribbon graphs of matrix models (dual to discretized surfaces), GFT graphs are dual to discretized three-dimensional topological spaces (not only manifolds but also more singular spaces). For the simplest GFT models [15], the Feynman amplitude of a graph equals the partition function of the discretized BF theory $[16,17] .{ }^{1}$ Naturally, the first

\footnotetext{
${ }^{1}$ More involved GFT models [18-21] have been proposed in an attempt to implement the Plebanski constraints and reproduce the gravity partition function.
} 
question one asks about tensor models and GFTs is if there exist some kind of $1 / N$ expansion generalizing the one of matrix models. Since their introduction [15] no conclusive result in this direction has been established. It is the most important challenge in the field today [22].

In this paper, we perform the complete $1 / N$ expansion of the "colored GFT" model [23-25] (CGFT). The terms in this expansion are indexed by "Core Graphs" encoding various topologies. Although somewhat abstract, the "Core Graphs" are a very convenient way to classify three-dimensional topological pseudo manifolds. The classification is not canonical, i.e. the same pseudo manifold is represented by multiple Core Graphs. Finding a complete, canonical, classification would amount to solving a very subtle and still open question in topology. At leading order in $1 / N$ only graphs dual to the three sphere $S^{3}$ contribute.

The $1 / N$ expansion we present uses estimates [26-30] of Feynman amplitudes in CGFT and results in combinatorial topology and manifold crystallization theory $[31,32]$. Almost none of the concepts and techniques we use can be applied to non-colored GFT models.

This paper is organized as follows. In Sect. 2, we recall the colored threedimensional Boulatov tensor model. Sections 3 and 4 introduce the techniques required to perform in Sect. 5 the $1 / N$ expansion of the model.

\section{The Colored Boulatov Model}

Let $G$ be some compact multiplicative Lie group and denote $h$ its elements, $e$ its unit, and $\int \mathrm{d} h$ the integral with respect to the Haar measure. Let $\bar{\psi}^{i}, \psi^{i}$, $i=0,1,2,3$ be four couples of complex scalar (or Grassmann) fields over three copies of $G, \psi^{i}: G \times G \times G \rightarrow \mathbb{C}$. We denote $\delta^{N}(h)$ the delta function over $G$ with some cutoff such that $\delta^{N}(e)$ is finite, but diverges (polynomially) when $N$ goes to infinity (further discussion on this point is presented in Sect. 6). For $G=S U(2)$ (denoting $\chi^{j}(h)$ the character of $h$ in the representation $j$ ) respectively $G=U(1)$ we can choose

$$
\left.\delta^{N}(h)\right|_{G=S U(2)}=\left.\sum_{j=0}^{N}(2 j+1) \chi^{j}(h) \quad \delta^{N}(\varphi)\right|_{G=U(1)}=\sum_{p=-N}^{N} e^{\imath p \varphi} .
$$

The partition function of the colored Boulatov model [23] over $G$ is the path integral

$$
Z(\lambda, \bar{\lambda})=e^{-F(\lambda, \bar{\lambda})}=\int \prod_{i=0}^{4} \mathrm{~d} \mu_{P}\left(\bar{\psi}^{i}, \psi^{i}\right) e^{-S^{\mathrm{int}}\left(\bar{\psi}^{i}, \psi^{i}\right)},
$$

with normalized Gaussian measure of covariance $P$

$$
\begin{aligned}
P_{h_{0} h_{1} h_{2} ; h_{0}^{\prime} h_{1}^{\prime} h_{2}^{\prime}} & =\int \mathrm{d} \mu_{P}\left(\bar{\psi}^{i}, \psi^{i}\right) \bar{\psi}_{h_{0} h_{1} h_{2}}^{i} \psi_{h_{0}^{\prime} h_{1}^{\prime} h_{2}^{\prime}}^{i} \\
& =\int \mathrm{d} h \delta^{N}\left(h_{0} h\left(h_{0}^{\prime}\right)^{-1}\right) \delta^{N}\left(h_{1} h\left(h_{1}^{\prime}\right)^{-1}\right) \delta^{N}\left(h_{2} h\left(h_{2}^{\prime}\right)^{-1}\right),
\end{aligned}
$$



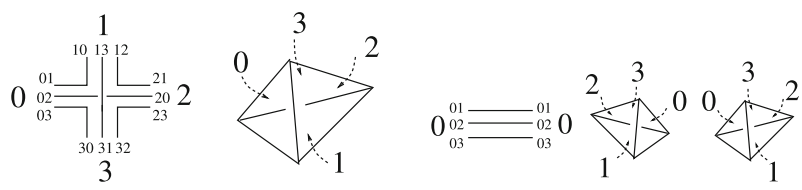

Figure 1. Colored GFT vertex and line
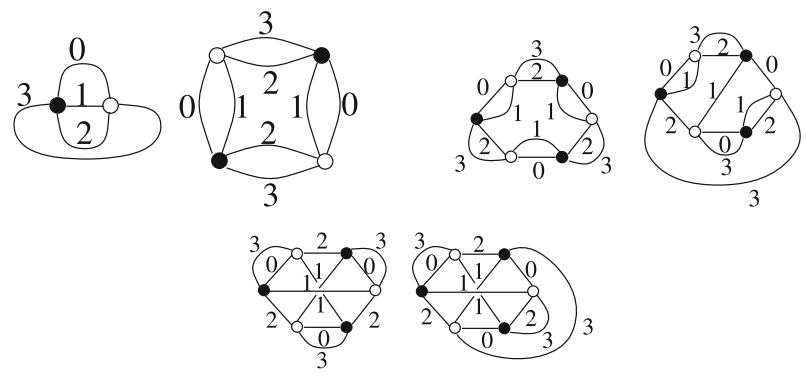

Figure 2. Examples of Colored GFT graphs

and interaction (denoting $\left.\psi(h, p, q)=\psi_{h p q}\right)$

$$
\begin{aligned}
S^{\text {int }}= & \frac{\lambda}{\sqrt{\delta^{N}(e)}} \int_{G^{6}} \psi_{h_{03} h_{02} h_{01}}^{0} \psi_{h_{10} h_{13} h_{12}}^{1} \psi_{h_{21} h_{20} h_{23}}^{2} \psi_{h_{32} h_{31} h_{30}}^{3} \\
& +\frac{\bar{\lambda}}{\sqrt{\delta^{N}(e)}} \int_{G^{6}} \bar{\psi}_{h^{03} h^{02} h^{01}}^{0} \bar{\psi}_{h^{10} h^{13} h^{12}}^{1} \bar{\psi}_{h^{21} h^{20} h^{23}}^{2} \bar{\psi}_{h^{32} h^{31} h^{30}}^{3}
\end{aligned}
$$

where $h_{i j}=h_{j i}$. We call "black" the vertex involving the $\psi$ 's and "white" the one involving the $\bar{\psi}$ 's.

The half lines of the CGFT vertex (represented in Fig. 1) have a color $i$. The group elements $h_{i j}$ in Eq. (4) are associated with the "strands" (represented as solid lines) common to the half lines $i$ and $j$. The vertex is dual to a tetrahedron, and its half lines represent the triangles bounding the tetrahedron. The strand $i j$, common to the half lines $i$ and $j$, represents the edge of the tetrahedron common to the triangles $i$ and $j$. The CGFT lines (Fig. 1) always connect two vertices of opposite orientation (i.e. a black and a white vertex). They have three parallel strands associated with the three arguments of the fields. A line represents the gluing of two tetrahedra (of opposite orientations) along triangles of the same color.

The strand structure of the vertex and propagator is fixed. One can represent a CGFT graph either as a stranded graph (using the vertex and propagator in Fig. 1) or as a "colored graph" with (colored) solid lines and two classes of oriented vertices. Some examples of CGFT graphs are given in Fig. 2. We denote them from left to right $\mathcal{G}_{1}, \mathcal{G}_{2}, \mathcal{G}_{3 ; a}, \mathcal{G}_{3 ; b}, \mathcal{G}_{3 ; c}$ and $\mathcal{G}_{3 ; d}$.

The lines of a vacuum CGFT graph $\mathcal{G}$ are oriented (say from the black to the white vertex). The closed strands of $\mathcal{G}$ form "faces" and are labeled by 
couples of colors. A vacuum CGFT graph must have the same number of black and white vertices. In this paper, we will only deal with connected graphs. We denote $\mathcal{N}_{\mathcal{G}}, \mathcal{L}_{\mathcal{G}}, \mathcal{F}_{\mathcal{G}}$ the sets of vertices, lines and faces of $\mathcal{G}$. Moreover, we denote $\mathcal{L}_{\mathcal{G}}^{i}$ the set of lines of color $i$ and $\mathcal{F}_{\mathcal{G}}^{i j}$ the set of faces of colors $i j$ of $\mathcal{G}$. The Feynman amplitude of $\mathcal{G}$ is

$$
A^{\mathcal{G}}=\frac{(\lambda \bar{\lambda})^{\frac{\mathcal{N}_{\mathcal{G}}}{2}}}{\left[\delta^{N}(e)\right]^{\frac{\left|\mathcal{N}_{\mathcal{G}}\right|}{2}}} \int \prod_{\ell \in \mathcal{L}_{\mathcal{G}}} \mathrm{d} h_{\ell} \prod_{f \in \mathcal{F}_{\mathcal{G}}} \delta_{f}^{N}\left(\prod_{\ell \in f} h_{\ell}^{\sigma^{\ell \mid f}}\right),
$$

where the notation $\ell \in f$ (which we sometimes omit) signifies that the line $\ell$ belongs to the face $f$ and $\sigma^{\ell \mid f}=1$ (resp. -1 ) if the orientations of $\ell$ and $f$ coincide (resp. are opposite). The $\delta^{N}$ functions are invariant under cyclic permutations and conjugation of their arguments; hence, the amplitude of a graph does not depend on the orientation of the faces or on their starting point.

The first ingredient in our $1 / N$ expansion is the scaling of the coupling in Eq. (4). In [28], it is proved that $A^{\mathcal{G}}$ obeys

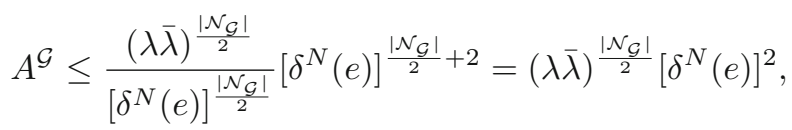

and that the bound is optimal (i.e. there exist graphs at any order saturating it). In order to obtain a sensible large $N$ limit, the scaling of the couplings $\lambda$ and $\bar{\lambda}$ must be chosen such that the maximally divergent graphs have uniform degree of divergence at all orders.

\section{Ribbon Graphs}

To any CGFT graph one associates two classes of ribbon graphs: its bubbles [23] and its jackets [29]. We denote in the sequel $\widehat{i}=\{0,1,2,3\} \backslash\{i\}$, $\widehat{i j}=\{0,1,2,3\} \backslash\{i, j\}$ and $\widehat{i j k}=\{0,1,2,3\} \backslash\{i, j, k\}$.

\subsection{Bubbles}

The bubbles [23] of a CGFT graph are the maximally connected subgraphs with three colors. They are dual to the vertices of the gluing of tetrahedra. ${ }^{2}$ The bubbles admit two representations, either as colored graphs or as ribbon graphs $[23,24]$. The ribbon graph of a bubble with colors $i, j, k$ is obtained by deleting all the strands containing the color $\widehat{i j k}$. The bubbles of the graph $\mathcal{G}_{1}$ (Fig. 2) are represented in Fig. 3. We denote $\mathcal{B}_{\mathcal{G}}$ the set of all the bubbles of $\mathcal{G}$ and $\mathcal{B}_{\mathcal{G}}^{i j k}$ the set of bubbles of colors $i j k$.

For a bubble $b \in \mathcal{B}_{\mathcal{G}}$, we denote $n_{b}, l_{b}$ and $f_{b}$ the sets of its vertices, lines and faces. The graph $\mathcal{G}$ has four valent vertices $\left(2\left|\mathcal{N}_{\mathcal{G}}\right|=\left|\mathcal{L}_{\mathcal{G}}\right|\right)$, while its

\footnotetext{
${ }^{2}$ Recently an alternative definition for bubbles has been proposed in [33]. Although interesting in itself, this definition is somewhat idiosyncratic, and it seems preferable to use the more standard notion of bubbles dual to vertices of the gluing of tetrahedra.
} 

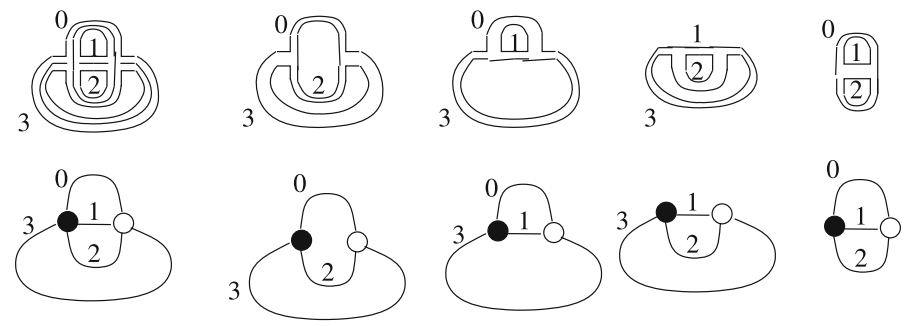

Figure 3. The bubbles of $\mathcal{G}_{1}$
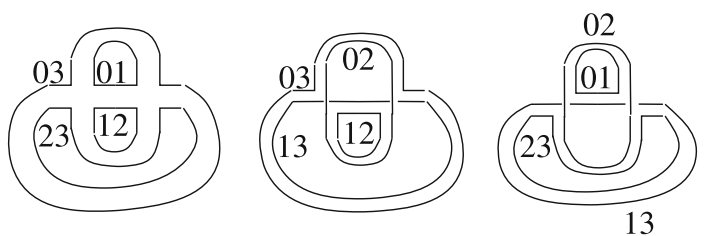

Figure 4. The jackets of $\mathcal{G}_{1}$

bubbles have three valent vertices $\left(3\left|n_{b}\right|=2\left|l_{b}\right|\right)$. We have

$$
\begin{aligned}
& 4\left|\mathcal{N}_{\mathcal{G}}\right|=\sum_{b \in \mathcal{B}_{\mathcal{G}}}\left|n_{b}\right|, \quad 3\left|\mathcal{L}_{\mathcal{G}}\right|=\sum_{b \in \mathcal{B}_{\mathcal{G}}}\left|l_{b}\right|, \quad 2\left|\mathcal{F}_{\mathcal{G}}\right|=\sum_{b \in \mathcal{B}_{\mathcal{G}}}\left|f_{b}\right|, \\
& \left|\mathcal{N}_{\mathcal{G}}\right|-\left|\mathcal{L}_{\mathcal{G}}\right|+\left|\mathcal{F}_{\mathcal{G}}\right|-\left|\mathcal{B}_{\mathcal{G}}\right|=-\sum_{b \in \mathcal{B}_{\mathcal{G}}} g_{b},
\end{aligned}
$$

with $g_{b}$ the genus of the bubble $b$. A graph $\mathcal{G}$ is dual to an orientable pseudo manifold. If all its bubbles are planar, then it is dual to an orientable manifold $[25]$.

\subsection{Jackets}

A second class of ribbon graphs associated with $\mathcal{G}$ are its jackets [29]. A jacket of $\mathcal{G}$ is the ribbon graph obtained from $\mathcal{G}$ by deleting all the faces with colors $i j$ and $\widehat{i j}$. A CGFT graph has three jackets. The three jackets of $\mathcal{G}_{1}$ are represented in Fig. 4, where the labels are associated with the faces.

The jackets of $\mathcal{G}$ have four valent ribbon vertices. The reader might be worried that while the vertices of the jacket with faces 02,13 deleted (the one originally identified in [29]) are simple ribbon vertices, the ones of the other two jackets (with the faces 03, 12 and 01,23 deleted) appear twisted in Fig. 4. This is just an illusion: permuting the half lines 0 and 1 and, respectively, 1 and 2 on every jacket vertex eliminates all the twists. The sets of vertices, lines and faces of a jacket are $\mathcal{N}_{\mathcal{G}}, \mathcal{L}_{\mathcal{G}}$ and $\mathcal{F}_{\mathcal{G}} \backslash \mathcal{F}_{\mathcal{G}}^{i j} \backslash \mathcal{F}_{\mathcal{G}}^{\hat{i j}}$.

\subsection{Face Routing}

In non-identically distributed matrix models [34-36], the amplitude of a Feynman graph is computed via a "routing" algorithm, a digested version of which we present below. 


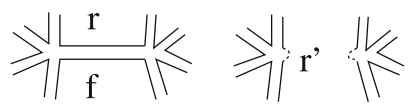

Figure 5. Deletion of a ribbon line

To every ribbon graph $\mathcal{H}$ (with sets of vertices, lines and faces denoted $\mathcal{N}, \mathcal{L}$ and $\mathcal{F}$ ), one associates a dual graph $\tilde{\mathcal{H}}$. The construction is standard (see for instance $[30,36]$ and references therein). The vertices of $\mathcal{H}$, correspond to the faces of $\tilde{\mathcal{H}}$, its lines to the lines of $\tilde{\mathcal{H}}$ and its faces to the vertices of $\tilde{\mathcal{H}}$. The lines of $\mathcal{H}$ admit (many) partitions in three disjoint sets: a tree $\mathcal{T}$ in $\mathcal{H}$, $(|\mathcal{T}|=|\mathcal{N}|-1)$, a tree $\tilde{\mathcal{T}}$ in the its dual $\tilde{\mathcal{H}},(|\tilde{\mathcal{T}}|=|\mathcal{F}|-1)$, and a set $\mathcal{L} \backslash \mathcal{T} \backslash \tilde{\mathcal{T}}$, $\left(|\mathcal{L} \backslash \mathcal{T} \backslash \tilde{\mathcal{T}}|=2 g_{\mathcal{H}}\right)$ of "genus" lines [30].

We orient the faces of $\mathcal{H}$ such that the two strands of every line have opposite orientations. We set a face of $\mathcal{H}$ as "root" (denoted $r$ ). Consider a face $f$ sharing some line $l(f, \tilde{\mathcal{T}}) \in \tilde{\mathcal{T}}$ with the root (i.e. the two strands of $l(f, \tilde{\mathcal{T}})$ belong one to $r$ and the other to $f)$. The group element $h_{l(f, \tilde{\mathcal{T}})}$ appears exactly once in the argument of $\delta_{f}^{N}$ and $\delta_{r}^{N}$

$$
\begin{aligned}
& \delta_{r}^{N}\left(\prod_{\ell} h_{\ell}^{\sigma^{\ell \mid r}}\right) \delta_{f}^{N}\left(\prod_{\ell} h_{\ell}^{\sigma^{\ell \mid f}}\right) \\
& \quad=\delta_{r}^{N}\left(\left(\underset{\ell \neq l(f, \tilde{\mathcal{T}})}{\overrightarrow{h_{\ell}}} h_{\ell}^{\sigma^{\ell \mid r}}\right) h_{l(f, \tilde{\mathcal{T}})}^{\sigma^{l(f, \tilde{\mid})}}\right) \delta_{f}^{N}\left(h_{l(f, \tilde{\mathcal{T}})}^{\sigma^{l(f \tilde{\mathcal{T}}) \mid f}}\left(\underset{\ell \neq l(f, \tilde{\mathcal{T}})}{\overrightarrow{\prod_{\ell}}} h_{\ell}^{\sigma^{\ell \mid f}}\right)\right),
\end{aligned}
$$

where we set $l(f, \tilde{\mathcal{T}})$ as the last line of $r$ and as the first line of $f$. By our choice of orientations $\sigma^{l(f, \tilde{\mathcal{T}}) \mid r} \sigma^{l(f, \tilde{\mathcal{T}}) \mid f}=-1$ and Eq. (8) becomes

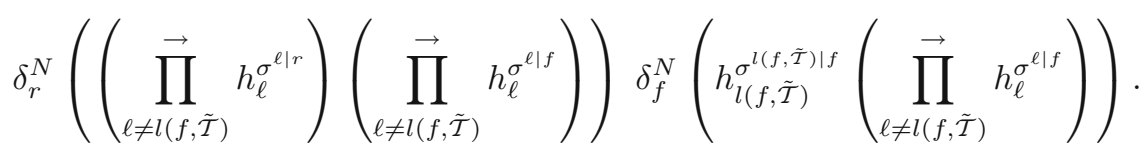

This trivial multiplication has two consequences. First, the face $f$ is canonically associated with the line $l(f, \tilde{\mathcal{T}})$. Second, the face $r$ becomes a root face in the graph $\mathcal{H}-l(f, \tilde{\mathcal{T}})$, obtained from $\mathcal{H}$ by deleting $l(f, \tilde{\mathcal{T}})$ and connecting $r$ and $f$ into a new face $r^{\prime}=r \cup f$ (see Fig. 5). Iterating for all faces except the root we get

$$
\begin{aligned}
& \prod_{f \in \mathcal{H}} \delta_{f}^{N}\left(\prod_{\ell} h_{\ell}^{\sigma^{\ell \mid f}}\right) \\
& \quad=\delta_{r}^{N}\left(\prod_{\ell \notin \tilde{\mathcal{T}}}^{\rightarrow} h_{\ell}^{\sigma^{\ell \mid \cup_{f} \in \mathcal{H}^{f}}}\right) \prod_{f \in \mathcal{H}, f \neq r} \delta_{f}^{N}\left(h_{l(f, \tilde{\mathcal{T}})}^{\sigma^{l(f, \tilde{\mathcal{T}}) \mid f}}\left(\prod_{\ell \neq l(f, \tilde{\mathcal{T}})}^{\overrightarrow{T^{\prime}}} h_{\ell}^{\sigma^{\ell \mid f}}\right)\right) .
\end{aligned}
$$

If $\mathcal{H}$ is planar, $\cup_{f \in \mathcal{H}} f$ is the exterior face of the tree $\mathcal{T}$ in $\mathcal{H}$. The group elements corresponding to lines of $\mathcal{T}$ touching leafs (vertices of coordination 
one in $\mathcal{T}$ ) appear consecutively $h_{l} h_{l}^{-1}$ and drop from the root face. Iterating for all line in $\mathcal{T}$ we get

$$
\left.\delta_{r}^{N}\left(\prod_{\ell \notin \tilde{\mathcal{T}}} h_{\ell}^{\sigma^{\ell \mid \cup} f \in \mathcal{H}^{f}}\right)\right|_{\mathcal{H} \text { planar }}=\delta^{N}(e),
$$

for any base group $G$. Remark that only the argument of the root $\delta_{r}^{N}$ changes under routing.

\subsection{An Example: Two-Dimensional GFT}

The two-dimensional GFT (with $G=S U(2)$ ) is a non-identically distributed matrix model. To see this, we develop the fields in Fourier modes

$$
\psi^{i}\left(g_{1}, g_{2}\right)=\sum \psi_{\left(j_{1}, m_{1}, n_{1}\right) ;\left(j_{2}, m_{2}, n_{2}\right)}^{i} \sqrt{d_{j_{1}} d_{j_{2}}} D_{m_{1} n_{1}}^{j_{1}}\left(g_{1}\right) \overline{D_{m_{2} n_{2}}^{j_{2}}\left(g_{2}\right)}
$$

where $d_{j}=2 j+1$. We denote $J_{1}=\left(j_{1}, m_{1}, n_{1}\right)$. In this normalization, the interaction is just a usual three-matrix interaction (the couplings do not need to be rescaled in this case)

$$
S^{\text {int }}=\lambda \psi_{J_{1} ; J_{2}}^{0} \psi_{J_{2} ; J_{3}}^{1} \psi_{J_{3}, J_{1}}^{2}+\bar{\lambda} \bar{\psi}_{J_{1} ; J_{2}}^{0} \bar{\psi}_{J_{2} ; J_{3}}^{1} \bar{\psi}_{J_{3}, J_{1}}^{2} \text {. }
$$

On the contrary, the two-point correlation function is (defining $\chi_{j}^{N}$ to be 1 if $j \leq N$ and 0 otherwise)

$$
\begin{aligned}
& \left\langle\bar{\psi}_{J_{1} ; J_{2}}^{i} \psi_{J_{1}^{\prime} ; J_{2}^{\prime}}^{i}\right\rangle=\sqrt{d_{j_{1}} d_{j_{2}} d_{j_{1}^{\prime}} d_{j_{2}^{\prime}}} \int D_{m_{1} n_{1}}^{j_{1}}\left(g_{1}\right) \\
& \quad \times \overline{D_{m_{2} n_{2}}^{j_{2}}\left(g_{2}\right)} \delta^{N}\left(g_{1} h\left(g_{1}^{\prime}\right)^{-1}\right) \delta^{N}\left(g_{2} h\left(g_{2}^{\prime}\right)^{-1}\right) \overline{D_{m_{1}^{\prime} n_{1}^{\prime}}^{j_{1}^{\prime}}\left(g_{1}^{\prime}\right)} D_{m_{2}^{\prime} n_{2}^{\prime}}^{j_{2}^{\prime}}\left(g_{2}^{\prime}\right),
\end{aligned}
$$

i.e., after performing the integrals over $g_{1}^{\prime}$ and $g_{2}^{\prime}$,

$$
\begin{aligned}
\sqrt{d_{j_{1}} d_{j_{2}} d_{j_{1}^{\prime}} d_{j_{2}^{\prime}}} \int D_{m_{1} n_{1}}^{j_{1}}\left(g_{1}\right) \overline{D_{m_{1}^{\prime} n_{1}^{\prime}}^{j_{1}^{\prime}}\left(g_{1} h\right)} \overline{D_{m_{2} n_{2}}^{j_{2}}\left(g_{2}\right)} D_{m_{2}^{\prime} n_{2}^{\prime}}^{j_{2}^{\prime}}\left(g_{2} h\right) \chi_{j_{1}^{\prime}}^{N} \chi_{j_{2}^{\prime}}^{N} \\
=\sqrt{d_{j_{1}} d_{j_{2}} d_{j_{1}^{\prime}} d_{j_{2}^{\prime}}} \sum_{k_{1}^{\prime}, k_{2}^{\prime}} \int D_{m_{1} n_{1}}^{j_{1}}\left(g_{1}\right) \overline{D_{m_{1}^{\prime} k_{1}^{\prime}}^{j_{1}^{\prime}}\left(g_{1}\right)} \overline{D_{k_{1}^{\prime} n_{1}^{\prime}}^{j_{1}^{\prime}}(h)} \\
=\frac{1}{D_{m_{2} n_{2}}^{j_{2}}\left(g_{2}\right)} D_{m_{2}^{\prime} k_{2}^{\prime}}^{j_{2}^{\prime}}\left(g_{2}\right) D_{k_{2}^{\prime} n_{2}^{\prime}}^{j_{2}^{\prime}}(h) \chi_{j_{1}^{\prime}}^{N} \chi_{j_{2}^{\prime}}^{N} \\
=\frac{1}{2 j_{1}+1} \delta^{j_{1}^{\prime} j_{2}^{\prime}} \delta^{j_{1}^{\prime} j_{1}} \delta_{2}^{j_{2}^{\prime} j_{2}} \sum_{k_{1}^{\prime}, k_{2}^{\prime}} \delta_{m_{1} m_{1}^{\prime}} \delta_{n_{1} k_{1}^{\prime}} \delta_{m_{2} m_{2}^{\prime}} \delta_{n_{2} k_{2}^{\prime}} \delta_{k_{1}^{\prime} k_{2}^{\prime}} \delta_{n_{1}^{\prime} n_{2}^{\prime}} \\
=\frac{\delta^{j_{1} j_{2}}}{2 j_{1}+1} \delta^{j_{1}^{\prime} j_{1}} \delta^{j_{2}^{\prime} j_{2}} \delta_{m_{1} m_{1}^{\prime}} \delta_{m_{2} m_{2}^{\prime}} \delta_{n_{1} n_{2}} \delta_{n_{1}^{\prime} n_{2}^{\prime}} \chi_{j_{1}^{\prime}}^{N} \chi_{j_{2}^{\prime}}^{N},
\end{aligned}
$$

which is certainly not identically distributed: not only the propagator has a weight proportional with $\frac{1}{2 j_{1}+1}$, but also it presents a twist in the indices $n .^{3}$

The free energy of the two-dimensional GFT admits a familiar "genus expansion". To see this, we expand it in Feynman graphs of amplitude given by Eq. 5 (where $\mathcal{G}$ is, in this case, just a simple ribbon graph). By face routing, one can integrate all group elements $h_{l}$ with $l \in \tilde{\mathcal{T}}$, and by a tree change of

${ }^{3}$ We thank an anonymous referee for pointing out the apparition of this twist. 

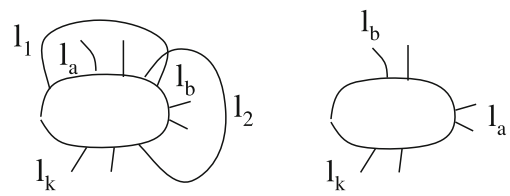

FiguRE 6. "Third Filk move" on a super rosette

variables [26], one eliminates all group elements $h_{l}$ with $l \in \mathcal{T}$. One is left with an integral over the genus lines corresponding to a "super rosette graph" $R_{g}$ with only one vertex and one face obtained from $\mathcal{G}$ by deleting the lines in $\tilde{\mathcal{T}}$ and contracting the lines in $\mathcal{T}$ [30]. Each genus line appears twice in the argument of the last $\delta^{N}$ function. To evaluate the amplitude of $R_{g}$, consider its dual super rosette $\tilde{R}_{g}$. The amplitude writes

$$
A^{R_{g}}=\int \prod_{\ell} \mathrm{d} h_{\ell} \delta^{N}\left(\vec{\prod} h_{\ell}^{\sigma_{\tilde{R}_{g}}^{\ell}}\right)
$$

where the product is taken along the (unique) vertex of $\tilde{R}_{g}$ and $\sigma_{\tilde{R}_{g}}^{\ell}$ is 1 if the line $\ell$ enters the vertex and -1 if it exits. A super rosette can be simplified by the "third Filk move" [30]. Consider two lines $\ell_{1}$ and $\ell_{2}$ forming a "nice crossing" on $\tilde{R}_{g}$, i.e. such that the end point of $\ell_{1}$ is the successor of the start point of $\ell_{2}$ (see Fig. 6). Denote the rest of the half lines encompassed by $\ell_{1}$ (resp. $\ell_{2}$ ) by $\ell_{a}$ (resp. $\ell_{b}$ ) and the remaining half lines $\ell_{k}$. If one deletes both $\ell_{1}$ and $\ell_{2}$ and permutes $\ell_{a}$ and $\ell_{b}$, the resulting graph $\left(\tilde{R}_{g}\right) / \ell_{1}, \ell_{2}$ will have only one vertex and one face (hence will be a super rosette) but two less lines (hence genus $g^{\prime}=g-1$ ). In this way, one can reduce all (pairs of) genus lines. Denoting $h_{a}=\prod h_{\ell_{a}}^{\sigma_{\tilde{R}_{g}}^{\ell_{a}}}, h_{b}=\prod h_{\ell_{b}}^{\sigma_{\tilde{R}_{g}}^{\ell_{b}}}$ and $h_{k}=\prod h_{\ell_{k}}^{\sigma_{\tilde{R}_{g}}^{\ell_{k}}}$, the contribution of $\ell_{1}$ and $\ell_{2}$ to the amplitude of $R_{g}$ writes

$$
\begin{aligned}
A^{R_{g}} & =\int \delta^{N}\left(h_{\ell_{1}} h_{a} h_{\ell_{2}} h_{\ell_{1}}^{-1} h_{b} h_{\ell_{2}}^{-1} h_{k}\right)=\sum_{j} d_{j} \int \chi^{j}\left(h_{\ell_{1}} h_{a} h_{\ell_{2}} h_{\ell_{1}}^{-1} h_{b} h_{\ell_{2}}^{-1} h_{k}\right) \\
& =\sum_{j} d_{j} \frac{1}{d_{j}^{2}} \int \chi^{j}\left(h_{a} h_{k} h_{b}\right),
\end{aligned}
$$

and the argument of $\chi^{j}$ corresponds to $\left(\tilde{R}_{g}\right) / \ell_{1}, \ell_{2}$. Iterating for all lines of the super rosette, we get $^{4}$

$$
A^{R_{g}}=\sum d_{j} \frac{1}{d_{j}^{2 g}} \chi^{j}(e)=\sum d_{j}^{2-2 g} \approx N^{3-2 g} .
$$

The particular super rosette to which a graph is reduced depends on the routing trees $\mathcal{T}$ and $\tilde{\mathcal{T}}$, but all super rosettes associated with a graph have the same genus $g$. One can define $\left[R_{g}\right]$ as the equivalence class of all super rosettes

4 To correctly identify the scaling with $N$ one must use sliced $\delta^{N}$ functions, $\delta^{N}(h)=$ $\sum_{N / 2}^{N} d_{j} \chi^{j}(h)$. 

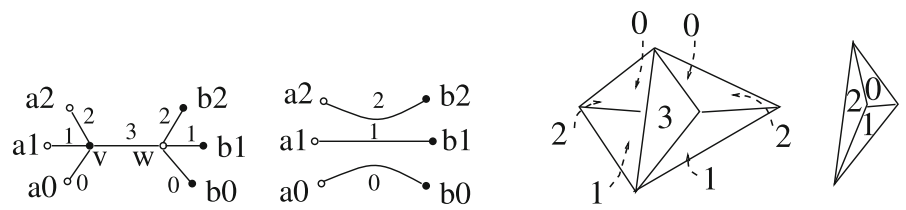

FiguRE 7. 1-Dipole contraction in $\mathcal{G}$ and its dual gluing

of genus $g$. The amplitude of $\mathcal{G}$ equals the one of the super rosette classes to which it belongs. The genus expansion of the free energy writes

$$
F(\lambda, \bar{\lambda})=\sum C^{\left[R_{g}\right]}(\lambda, \bar{\lambda}) A^{\left[R_{g}\right]}=\sum C^{\left[R_{g}\right]}(\lambda, \bar{\lambda}) N^{3-2 g},
$$

with $C^{\left[R_{g}\right]}(\lambda, \bar{\lambda})$ a combinatorial factor counting the graphs which reduce to the super rosette class $\left[R_{g}\right]$, i.e. all graphs of genus $g$. Of course in two dimensions, as the super rosette amplitudes can be computed explicitly one completely forgets about them, indexes the expansion of the free energy by the genus $g$ and concludes that higher and higher genus graphs are suppressed by larger and larger powers of the cut off.

\section{Dipoles}

The second ingredient we need to establish our results are the Dipole moves $[31,32]$ encoding homeomorphisms of pseudo manifolds (we will make a precise statement later). We will identify the various bubbles, faces and lines below by their colors (in superscript) and their vertices (in subscript).

\subsection{1-Dipoles}

Consider a line of color 3 with end vertices $v$ and $w$ (denoted $L_{v w}^{3}$ ) in a graph $\mathcal{G}$. Call $a_{0}\left(a_{1}\right.$ and $\left.a_{2}\right)$ the end vertex of the line of color 0 (1 and 2) touching $v$, and $b_{0}\left(b_{1}\right.$ and $\left.b_{2}\right)$ the end vertex of the line of color 0 ( 1 and 2$)$ touching $w$ (see Fig. 7). The vertices $v$ and $w$ belong each to some three bubbles of colors $012, B_{v a_{0} a_{1} a_{2}}^{012}$ and $B_{w b_{0} b_{1} b_{2}}^{012}$. The two bubbles might coincide or might be different. If they are different and at least one of them is planar, then the line $L_{v w}^{3}$ is called a 1 -Dipole.

A 1-Dipole can be contracted, i.e. the line $L_{v w}^{3}$ together with the vertices $v$ and $w$ can be deleted from the graph, and the remaining lines reconnected respecting the coloring (see Fig. 7). In the dual gluing, a 1-Dipole of color 3 represents two tetrahedra sharing the triangle (of color 3) such that the vertices opposite to the triangle (duals to $B_{v a_{0} a_{1} a_{2}}^{012}$ and $B_{w b_{0} b_{1} b_{2}}^{012}$ ) are different. The contraction translates in squashing the two tetrahedra, merging the two vertices, and coherently identifying the remaining triangles 0,1 and 2 (see Fig. 7).

In this picture, it is clear why one of $B_{v a_{0} a_{1} a_{2}}^{012}$ or $B_{w b_{0} b_{1} b_{2}}^{012}$ is required to be planar. If both points opposite to the triangle 3 were isolate singularities, the squashing of tetrahedra would decrease the number of singular points and 
would not be a homeomorphism. It is however a homeomorphism as long as one of the points is regular. ${ }^{5}$

The vertices $v$ and $w$ belong to the same faces 03,13 and $23\left(F_{v w a_{0} b_{0}}^{03}\right.$, $\left.F_{v w a_{1} b_{1}}^{13}, F_{v w a_{2} b_{2}}^{23}\right)$, but distinct faces 01,02 and $12\left(F_{v a_{0} a_{1}}^{01}, F_{w b_{0} b_{1}}^{01}, F_{v a_{0} a_{2}}^{02}\right.$, $F_{w b_{0} b_{2}}^{02}$ and $\left.F_{v a_{1} a_{2}}^{12}, F_{w b_{2} b_{2}}^{12}\right)$. They also belong to the same bubbles 013, 023 and 123, $\left(B_{v w a_{0} a_{1} b_{0} b_{1}}^{013}, B_{v w a_{0} a_{2} b_{0} b_{2}}^{023}, B_{v w a_{1} a_{2} b_{1} b_{2}}^{123}\right)$ but different bubbles 012 $\left(B_{v a_{0} a_{1} a_{2}}^{012}\right.$ and $\left.B_{w b_{0} b_{1} b_{2}}^{012}\right)$. We track the effect of the 1-Dipole contraction on the graph $\mathcal{G}$. Taking $B_{v a_{0} a_{1} a_{2}}^{012}$ the planar bubble, the contraction

- deletes the vertices $v$ and $w$ and the line $L_{v w}^{3}$.

- glues $L_{v a_{0}}^{0}$ on $L_{w b_{0}}^{0}$ to form a new line $L_{v w}^{0}$ (and similarly for colors 1 and 2).

- transforms the face $F_{v w a_{0} b_{0}}^{03}$ into a face $F_{a_{0} b_{0}}^{03}$ (and similarly for 13 and 23).

- glues the face $F_{v a_{0} a_{1}}^{01}$ on the face $F_{w b_{0} b_{1}}^{01}$ to form a new face $F_{a_{0} b_{0} b_{1} a_{1}}^{01}$ (and similarly for 02 and 12 ).

- transforms the bubble $B_{v w a_{0} a_{1} b_{0} b_{1}}^{013}$ into a bubble $B_{a_{0} a_{1} b_{0} b_{1}}^{013}$ (and similarly for 023 and 123)

- glues $B_{v a_{0} a_{1} a_{2}}^{012}$ on $B_{w b_{0} b_{1} b_{2}}^{012}$ to form a new bubble $B_{a_{0} b_{0} a_{1} b_{1} a_{2} b_{2}}^{012}$.

The bubbles 013, 023 and 123 transform trivially under contraction. Call $n, l, f$ and $g\left(n^{\prime}, l^{\prime}, f^{\prime}\right.$ and $\left.g^{\prime}\right)$ the vertices, lines, faces and genus of one of these bubbles before (after) contraction. We have

$$
\left|n^{\prime}\right|=|n|-2, \quad\left|l^{\prime}\right|=|l|-3, \quad\left|f^{\prime}\right|=|f|-1 \Rightarrow g^{\prime}=g .
$$

The bubble $B_{v a_{0} a_{1} a_{2}}^{012}$ (with $n_{a}, l_{a} f_{a}$ and $g_{a}$ ) is glued on $B_{w b_{0} b_{1} b_{2}}^{012}$ (with $n_{b}, l_{b} f_{b}$ and $\left.g_{b}\right)$ to form the new bubble $B_{a_{0} b_{0} a_{1} b_{1} a_{2} b_{2}}^{012}$ (with $n_{b}^{\prime}, l_{b}^{\prime}, f_{b}^{\prime}$ and $g_{b}^{\prime}$ ) and

$\left|n_{b}^{\prime}\right|=\left|n_{a}\right|+\left|n_{b}\right|-2,\left|l_{b}^{\prime}\right|=\left|l_{a}\right|+\left|l_{b}\right|-3,\left|f_{b}^{\prime}\right|=\left|f_{a}\right|+\left|f_{b}\right|-3 \Rightarrow g_{b}^{\prime}=g_{a}+g_{b}$.

Thus, $g_{b}^{\prime}=g_{b}$ if $g_{a}=0$. If $B_{w b_{0} b_{1} b_{2}}^{012}$ is dual to a conical singularity $\left(g_{b} \neq 0\right)$, then the new bubble $B_{a_{0} b_{0} a_{1} b_{1} a_{2} b_{2}}^{012}$ is dual to an identical singularity, and the two dual pseudo manifolds are homeomorphic [32]. Were we to allow a contraction when both $g_{a}, g_{b} \neq 0$ we would merge two conical singularities into a unique (more degenerate) conical singularity.

\subsection{Amplitude}

Suppose that all lines enter $v$ and exit $w$. We denote $h_{0 ; v}$ the group element associated with $L_{v a_{0}}^{0}$, etc. and use the shorthand notation $(01) ; v$ for $F_{v a_{0} a_{1}}^{01}$ etc. The contribution of all faces containing $v$ and/or $w$ to the amplitude of $\mathcal{G}$ is

\footnotetext{
5 See [32], especially the remark on page 93 in the proof of the main theorem.
} 


$$
\begin{aligned}
& \int \mathrm{d} h_{0 ; v} \mathrm{~d} h_{0 ; w} \mathrm{~d} h_{1 ; v} \mathrm{~d} h_{1 ; w} \mathrm{~d} h_{2 ; v} \mathrm{~d} h_{2 ; w} \mathrm{~d} h_{3} \\
& \delta_{(03)}^{N}\left(h_{0 ; v} h_{3}^{-1} h_{0 ; w} f^{03}\right) \delta_{(13)}^{N}\left(h_{1 ; v} h_{3}^{-1} h_{1 ; w} f^{13}\right) \delta_{(23)}^{N}\left(h_{2 ; v} h_{3}^{-1} h_{2 ; w} f^{23}\right) \\
& \delta_{(01) ; v}^{N}\left(h_{0 ; v} h_{1 ; v}^{-1} f_{v}^{01}\right) \delta_{(02) ; v}^{N}\left(h_{2 ; v} h_{0 ; v}^{-1} f_{v}^{02}\right) \delta_{(12) ; v}^{N}\left(h_{1 ; v} h_{2 ; v}^{-1} f_{v}^{12}\right) \\
& \quad \delta_{(01) ; w}^{N}\left(h_{1 ; w}^{-1} h_{0 ; w} f_{w}^{01}\right) \delta_{(02) ; w}^{N}\left(h_{0 ; w}^{-1} h_{2 ; w} f_{w}^{02}\right) \delta_{(12) ; w}^{N}\left(h_{2 ; w}^{-1} h_{1 ; w} f_{w}^{12}\right),
\end{aligned}
$$

where $f^{03}$ denotes the product of the remaining group elements along the face 03 and similarly for the rest. We first change variables to $h_{0 ; w}^{\prime}=h_{3}^{-1} h_{0 ; w}$, $d h_{0 ; w}^{\prime}=d h_{0 ; w}$ (and similarly for $h_{1 ; w}$ and $h_{2 ; w}$ ). The integral over $h_{3}$ is trivial. Forgetting the primes we obtain

$$
\begin{aligned}
& \int \mathrm{d} h_{0 ; v} \mathrm{~d} h_{0 ; w} \mathrm{~d} h_{1 ; v} \mathrm{~d} h_{1 ; w} \mathrm{~d} h_{2 ; v} \mathrm{~d} h_{2 ; w} \\
& \quad \delta_{(03)}^{N}\left(h_{0 ; v} h_{0 ; w} f^{03}\right) \delta_{(13)}^{N}\left(h_{1 ; v} h_{1 ; w} f^{13}\right) \delta_{(23)}^{N}\left(h_{2 ; v} h_{2 ; w} f^{23}\right) \\
& \delta_{(01) ; v}^{N}\left(h_{0 ; v} h_{1 ; v}^{-1} f_{v}^{01}\right) \delta_{(02) ; v}^{N}\left(h_{2 ; v} h_{0 ; v}^{-1} f_{v}^{02}\right) \delta_{(12) ; v}^{N}\left(h_{1 ; v} h_{2 ; v}^{-1} f_{v}^{12}\right) \\
& \quad \delta_{(01) ; w}^{N}\left(h_{1 ; w}^{-1} h_{0 ; w} f_{w}^{01}\right) \delta_{(02) ; w}^{N}\left(h_{0 ; w}^{-1} h_{2 ; w} f_{w}^{(02)}\right) \delta_{(12) ; w}^{N}\left(h_{2 ; w}^{-1} h_{1 ; w} f_{w}^{(12)}\right) .
\end{aligned}
$$

We change again variables to $h_{0}=h_{0 ; v} h_{0 ; w} \mathrm{~d} h_{0}=h_{0 ; w}$ (and similarly for $h_{1 ; w}$ and $\left.h_{2 ; w}\right)$ to obtain

$$
\begin{aligned}
& \int \mathrm{d} h_{0 ; v} \mathrm{~d} h_{0} \mathrm{~d} h_{1 ; v} \mathrm{~d} h_{1} \mathrm{~d} h_{2 ; v} \mathrm{~d} h_{2} \\
& \delta_{(03)}^{N}\left(h_{0} f^{03}\right) \delta_{(13)}^{N}\left(h_{1} f^{13}\right) \delta_{(23)}^{N}\left(h_{2} f^{23}\right) \\
& \delta_{(01) ; v}^{N}\left(h_{0 ; v} h_{1 ; v}^{-1} f_{v}^{01}\right) \delta_{(02) ; v}^{N}\left(h_{2 ; v} h_{0 ; v}^{-1} f_{v}^{02}\right) \delta_{(12) ; v}^{N}\left(h_{1 ; v} h_{2 ; v}^{-1} f_{v}^{12}\right) \\
& \delta_{(01) ; w}^{N}\left(h_{1}^{-1} h_{1 ; v} h_{0 ; v}^{-1} h_{0} f_{w}^{01}\right) \delta_{(02) ; w}^{N}\left(h_{0}^{-1} h_{0 ; v} h_{2 ; v}^{-1} h_{2} f_{w}^{02}\right) \\
& \quad \delta_{(12) ; w}^{N}\left(h_{2}^{-1} h_{2 ; v} h_{1 ; v}^{-1} h_{1} f_{w}^{12}\right) .
\end{aligned}
$$

We integrate $h_{1 ; v}, h_{2 ; v}$ using $\delta_{(01) ; v}^{N}$ and $\delta_{(02) ; v}^{N}$ (hence $h_{1 ; v}=f_{v}^{01} h_{0 ; v}, h_{2 ; v}^{-1}=$ $\left.h_{0 ; v}^{-1} f_{v}^{02}\right)$, and Eq. (22) becomes

$$
\begin{aligned}
& \int \mathrm{d} h_{0 ; v} \mathrm{~d} h_{0} \mathrm{~d} h_{1} \mathrm{~d} h_{2} \\
& \quad \delta_{(03)}^{N}\left(h_{0} f^{03}\right) \delta_{(13)}^{N}\left(h_{1} f^{13}\right) \delta_{(23)}^{N}\left(h_{2} f^{23}\right) \\
& \quad \delta_{(12) ; v}^{N}\left(f_{v}^{01} f_{v}^{02} f_{v}^{12}\right) \\
& \quad \delta_{(01) ; w}^{N}\left(h_{1}^{-1} f_{v}^{01} h_{0} f_{w}^{01}\right) \delta_{(02) ; w}^{N}\left(h_{0}^{-1} f_{v}^{02} h_{2} f_{w}^{02}\right) \delta_{(12) ; w}^{N}\left(h_{2}^{-1} f_{v}^{12} h_{1} f_{w}^{12}\right) .
\end{aligned}
$$

Remark that, ignoring $\delta_{(12) ; v}^{N}$, the integrand of Eq. (25) corresponds to the graph with the 1-Dipole contracted. But $\delta_{(12) ; v}^{N}$ reproduces the external face of a ribbon graph obtained by cutting the vertex $v$ in the bubble $B_{v a_{0} a_{1} a_{2}}^{012}$. The latter is a planar ribbon graph; hence, by Eq. (11) $\delta_{(12) ; v}^{N}\left(f_{v}^{01} f_{v}^{02} f_{v}^{12}\right)$ can be replaced by $\delta^{N}(e)$. Recalling that the number of vertices decreases by 2 we obtain that the amplitudes of $\mathcal{G}$ and $\mathcal{G}-L_{v w}^{3}$ (the graph with the 1-Dipole 


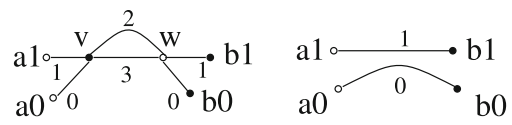

FigURE 8. 2-Dipole contraction

$L_{v w}^{3}$ contracted) are proportional

$$
A^{\mathcal{G}}=\frac{(\lambda \bar{\lambda})}{\delta^{N}(e)} \delta^{N}(e) A^{\mathcal{G}-L_{v w}^{3}}=(\lambda \bar{\lambda}) A^{\mathcal{G}-L_{v w}^{3}} .
$$

\subsection{2-Dipoles}

A 2-Dipole of colors 23 (see Fig. 8) is a couple of lines connecting the same two vertices $v$ and $w, L_{v w}^{2}$ and $L_{v w}^{3}$ such that the faces $F_{v a_{0} a_{1}}^{01}$ and $F_{w b_{0} b_{1}}^{01}$ are different. The 2-Dipole forms a face $F_{v w}^{23}$. Like the 1-Dipoles, the 2-Dipoles can be contracted (by deleting the lines 2 and 3 forming the 2-Dipole and reconnecting the rest of the lines respecting the colors). This is represented in Fig. 8. After contraction, the two faces $F_{v a_{0} a_{1}}^{01}$ and $F_{w b_{0} b_{1}}^{01}$ are glued into a unique face $F_{a_{0} a_{1} b_{0} b_{1}}^{01}$. A 2-Dipole is dual to two tetrahedra sharing two triangles (of colors 2 and 3 for Fig. 8) such that the edge opposite to the two triangles in each tetrahedron (dual to the faces $F_{v a_{0} a_{1}}^{01}$ and $F_{w b_{0} b_{1}}^{01}$ ) are different. The contraction translates in squashing the two tetrahedra and coherently identifying the remaining boundary triangles. This move always represents a homeomorphism [32]. Denoting $\mathcal{G}-F_{v w}^{23}$ the graph obtained from $\mathcal{G}$ after contracting the 2-Dipole, a short computation along the lines of the one for 1-Dipoles yields

$$
A^{\mathcal{G}}=\frac{(\lambda \bar{\lambda})}{\delta^{N}(e)} A^{\mathcal{G}-F_{v w}^{23}} .
$$

The Dipole contraction moves can be inverted into Dipole creation moves. The fundamental result we will use in the sequel [32] is that two pseudo manifolds dual to colored graphs $\mathcal{G}$ and $\mathcal{G}^{\prime}$ are homeomorphic if $\mathcal{G}$ and $\mathcal{G}^{\prime}$ are related by a finite sequence of 1 and 2-Dipole creation and contraction moves. We call two such graphs "equivalent", $\mathcal{G} \sim \mathcal{G}^{\prime}$.

\section{Bubble Routing and Core Graphs}

In the literature one finds two classes of results (bounds and evaluations) for amplitudes of GFT graphs. They are expressed either in terms of the number of vertices $[27,28]$ or in terms of the number of bubbles $[26,29]$. In order to build the $1 / N$ expansion in CGFT, we need to strike the right balance between the vertices and the bubbles of a graph. This is achieved by a bubble routing algorithm.

\subsection{Bubble Routing}

We start by choosing a set of roots of $\mathcal{G}$ for all colors $i$. For the color 3 , if all the bubbles $\mathcal{B}^{012}$ are planar, we choose one of them as root and denote it $R_{1}^{012}$. If there exist non-planar bubbles 012 , we set a non-planar bubble as principal 


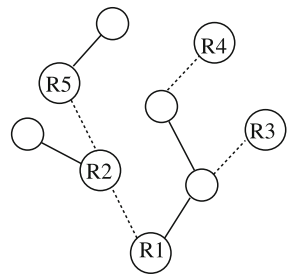

R5

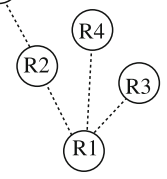

Figure 9. A tree $\mathcal{T}^{3}$ in the 012 connectivity graph

root $R_{1}^{012}$, and the other non-planar bubbles as "branch roots" $R_{2}^{012}, R_{3}^{012}, \ldots$. We denote the set of 012 roots of $\mathcal{G}$ by $\mathcal{R}^{012}=\left\{R_{1}^{012}, R_{2}^{012}, \ldots\right\}$. We repeat this for all colors (and denote $\mathcal{R}_{\mathcal{G}}$ the set of all roots of $\mathcal{G}$ ).

We associate with the bubbles 012 of $\mathcal{G}$ a "012 connectivity graph". Its vertices represent the various bubbles 012. Its lines are the lines of color 3 in $\mathcal{G}$. They either start and end on the same bubble 012 (in which case they are "tadpole" lines in the connectivity graph), or not. A particularly simple way to picture the 012 connectivity graph is to draw $\mathcal{G}$ with lines 0,1 and 2 much shorter than the lines 3 . We chose a tree in the connectivity graph, $\mathcal{T}^{3}$ (and call the rest of the lines 3 "loop lines"). For a branch root $R_{q}^{012}$, the line incident on it and belonging to the path in $\mathcal{T}^{3}$ connecting $R_{q}^{012}$ to the principal root $R_{1}^{012}$ is represented as dashed. All the other lines in $\mathcal{T}^{3}$ are represented as solid lines. An example is given in Fig. 9.

All the solid lines in $\mathcal{T}^{3}$ are 1-Dipoles, and we contract them. We end up with a connectivity graph with vertices corresponding to the roots $R_{q}^{012}$. The remaining lines of color 3 cannot be 1-Dipoles (they are either tadpole lines or they separate two non-planar roots). The number of 1-Dipoles of color 3 contracted is $\left|\mathcal{B}^{012}\right|-\left|\mathcal{R}^{012}\right|$. Neither the number nor the topology of the bubbles of the other colors $B^{013}, B^{023}$ and $B^{123}$ is changed under these contractions.

Having exhausted a complete set of 1-Dipoles of color 3 , we repeat the procedure for the 1-Dipoles of color 2. The routing tree $\mathcal{T}^{2}$ is chosen in the graph obtained after contracting the 1-Dipoles of color 3 and depends on $\mathcal{T}^{3}$, $\mathcal{T}^{2}\left(\mathcal{T}^{3}\right)$. The contraction of 1 -Dipole of color 2 changes the 012 connectivity graph, but it cannot create new 1-Dipoles of color 3: the topology of the 012 bubbles is unaffected by reducing 1-Dipoles of color 2; hence, the lines of color 3 will still either be tadpole lines or separate two non-planar roots 012 . After a full set of 1-Dipole contractions indexed by four distinct routing trees $\mathcal{T}^{3}, \mathcal{T}^{2}\left(\mathcal{T}^{3}\right), \mathcal{T}^{1}\left(\mathcal{T}^{2}, \mathcal{T}^{3}\right), \mathcal{T}^{0}\left(\mathcal{T}^{1}, \mathcal{T}^{2}, \mathcal{T}^{3}\right)$, we obtain a Core Graph. ${ }^{6}$

Definition 1 (Core Graph). A colored graph with $2 p$ vertices $\mathcal{G}_{p}$ is called a Core Graph at order $p$ if, for all colors $i$, it either has a unique (planar or non-planar) bubble $\widehat{P_{1}^{\hat{i}}}$ or all its bubbles $\widehat{P_{1}^{i}}, \widehat{P_{2}^{i}}, \ldots$ are non-planar.

\footnotetext{
${ }^{6}$ If $\mathcal{G}$ is dual to a manifold and one further reduces a full set of 2-Dipoles, one recovers a "gem" graph of [31].
} 
The amplitude of the graph $\mathcal{G}$ and of the Core Graph obtained after routing are related by

$$
A^{\mathcal{G}}=(\lambda \bar{\lambda})^{\left|\mathcal{B}_{\mathcal{G}}\right|-\left|\mathcal{R}_{\mathcal{G}}\right|} A^{\mathcal{G}_{p}}, \quad 2 p=\left|\mathcal{N}_{\mathcal{G}}\right|-2\left(\left|\mathcal{B}_{\mathcal{G}}\right|-\left|\mathcal{R}_{\mathcal{G}}\right|\right) .
$$

The Core Graph one obtains by routing is not independent of the routing trees $\mathcal{T}^{3}, \mathcal{T}^{2}, \mathcal{T}^{1}, \mathcal{T}^{0}$. The same graph leads to several equivalent Core Graphs, all at the same order $p, \mathcal{G}_{p} \sim \mathcal{G}_{p}^{\prime} \sim \ldots$. One can prove that all equivalent Core Graphs at the same order $\mathcal{G}_{p} \sim \mathcal{G}_{p}^{\prime}$ have the same amplitude. Only the creation/contraction of dipoles of color $i$ can change the number of bubbles of colors $\hat{i}$, and the latter only create/annihilate planar bubbles. It follows that the numbers of bubbles of colors $\widehat{i}$ of $\mathcal{G}_{p}$ and $\mathcal{G}_{p^{\prime}}$ are equal, and consequently, the total numbers of 1-Dipole creations and contractions are equal. As $\mathcal{G}_{p}$ and $\mathcal{G}_{p}^{\prime}$ have the same number of vertices, the total numbers of 2-Dipole creations and contractions are also equal and $A^{\mathcal{G}_{p}}=A^{\mathcal{G}_{p}^{\prime}}$.

We denote $\mathfrak{G}_{p}=\left\{\left[\mathcal{G}_{p}\right]\right\}$ the set of equivalence classes of Core Graphs at order $p$ under the equivalence relation $\sim$. The amplitude is a well-defined function of the equivalence class $\left[\mathcal{G}_{p}\right]$. Under an arbitrary routing, any graph will fall in a unique equivalence class $\left[\mathcal{G}_{p}\right]$. The free energy of the colored Boulatov model admits a topological expansion in Core Graphs classes

$$
F(\lambda, \bar{\lambda})=\sum_{p=1}^{\infty} \sum_{\left[\mathcal{G}_{p}\right] \in \mathfrak{G}_{p}} C^{\left[\mathcal{G}_{p}\right]}(\lambda, \bar{\lambda}) A^{\left[\mathcal{G}_{p}\right]},
$$

where $C^{\left[\mathcal{G}_{p}\right]}(\lambda, \bar{\lambda})$ is a combinatorial factor counting all the graphs routing to a Core Graph class at order $p$. The scaling with $N$ is entirely captured by the Core Graph amplitude $A^{\left[\mathcal{G}_{p}\right]}$. A Core Graphs class is dual to a specific pseudo manifold. Note however that the same pseudo manifold is represented by an infinity of classes $\left[\mathcal{G}_{p}\right]$ at higher and higher orders in $p$.

Core Graphs are in three dimensions, the appropriate generalization of the super rosettes of two-dimensional GFT. The only ingredient missing at this point is some estimate of their amplitude.

Theorem 1 (The Core Graph bound). The amplitude of a Core Graph at order $p, \mathcal{G}_{p}$, with set of bubble $\mathcal{P}$ respects

$$
\left|A^{\mathcal{G}_{p}}\right| \leq(\lambda \bar{\lambda})^{p}\left[\delta^{N}(e)\right]^{-\frac{1}{3} p+\frac{1}{3} \sum_{b \in \mathcal{P}}\left(1-g_{b}\right)+1} .
$$

Proof. We denote the set of lines and faces of $\mathcal{G}_{p}$ by $\mathcal{L}$ and $\mathcal{F}$. The amplitude of the Core Graph is

$$
A^{\mathcal{G}_{p}}=\frac{(\lambda \bar{\lambda})^{p}}{\left[\delta^{N}(e)\right]^{p}} \int \prod_{\ell \in \mathcal{L}} \mathrm{d} h_{\ell} \prod_{f \in \mathcal{F}} \delta_{f}^{N}\left(\prod_{\ell \in f} h_{\ell}^{\sigma^{\ell \mid f}}\right) .
$$

Denote $\mathcal{J}^{i j}$ the jacket of $\mathcal{G}_{p}$ with the faces $i j$ and $\widehat{i j}$ deleted. The idea is to use the jacket graph to integrate explicitly as many group elements as possible. Indeed, routing the faces of the jacket graph will associate a line with all (save one) of its faces. Integrating, all (save one) of the $\delta^{N}$ functions of the faces of the jacket graph will contribute 1 , as $\int \mathrm{d} h \delta^{N}\left(h^{-1} \ldots\right) K(h)=K(\ldots)$. 
The effect of these integrations over the rest of the $\delta^{N}$ functions is exceedingly complicated to track. However, we will not need to do it as we will just use a naive bound $\delta^{N}(h) \leq \delta^{N}(e)$ for all of them. In detail,

$$
A^{\mathcal{G}_{p}}=\frac{(\lambda \bar{\lambda})^{p}}{\left[\delta^{N}(e)\right]^{p}} \int \prod_{\ell \in \mathcal{L}} \mathrm{d} h_{\ell}\left[\prod_{f^{\prime} \in \mathcal{F}^{i j} \cup \mathcal{F}^{\widehat{i j}}} \delta_{f^{\prime}}^{N}\left(\prod_{\ell \in f^{\prime}} h_{\ell}^{\sigma^{\ell \mid f^{\prime}}}\right)\right]\left[\prod_{f \in \mathcal{J}^{i j}} \delta_{f}^{N}\left(\prod_{\ell \in f}^{\overrightarrow{h^{\prime}}} h_{\ell}^{\sigma^{\ell \mid f}}\right)\right],
$$

and routing the faces of the jacket graph via a tree $\tilde{\mathcal{T}}$ in the dual graph of the jacket we get

$$
\begin{aligned}
A^{\mathcal{G}_{p}}= & \frac{(\lambda \bar{\lambda})^{p}}{\left[\delta^{N}(e)\right]^{p}} \int \prod_{\ell \in \mathcal{L}_{p}} \mathrm{~d} h_{\ell}\left[\prod_{f^{\prime} \in \mathcal{F}^{i j} \cup \mathcal{F}^{\widehat{i j}}} \delta_{f}^{N}\left(\prod_{\ell \in f^{\prime}} h_{\ell}^{\sigma^{\ell \mid f^{\prime}}}\right)\right] \\
& \times\left[\delta_{r}^{N}\left(\prod_{\ell \notin \tilde{\mathcal{T}}}^{\rightarrow} h_{\ell}^{\sigma^{\ell \mid \cup_{f} \in \mathcal{J}^{i j} f}}\right) \prod_{f \in \mathcal{J}^{i j}, f \neq r} \delta_{f}^{N}\left(h_{l(f, \tilde{\mathcal{T}})}^{\sigma^{l(f, \tilde{\mathcal{T}}) \mid f}}\left(\prod_{\ell \neq l(f, \tilde{\mathcal{T}})}^{\rightarrow} h_{\ell}^{\sigma^{\ell \mid f}}\right)\right)\right] .
\end{aligned}
$$

Each of the $\delta^{N}$ of the faces of the jacket can now be associated uniquely with a specific integral over some group element. For all the lines in $\tilde{\mathcal{T}}$, we change variables to

$$
\tilde{h}_{l(f, \tilde{\mathcal{T}})}=h_{l(f, \tilde{\mathcal{T}})}^{\sigma^{l(f, \tilde{\mathcal{T}}) \mid f}}\left(\prod_{\ell \neq l(f, \tilde{\mathcal{T}})}^{\rightarrow} h_{\ell}^{\sigma^{\ell \mid f}}\right)
$$

and write (in sloppy notations)

$$
\begin{aligned}
A^{\mathcal{G}_{p}}= & \frac{(\lambda \bar{\lambda})^{p}}{\left[\delta^{N}(e)\right]^{p}} \int \prod_{\ell \in \mathcal{L}_{p} \backslash \tilde{\mathcal{T}}} \mathrm{d} h_{\ell} \prod_{l \in \tilde{\mathcal{T}}} \mathrm{d} \tilde{h}_{l}\left[\prod_{f^{\prime} \in \mathcal{F}_{p}^{i j} \cup \mathcal{F}_{p}^{\widehat{i j}}} \delta_{f^{\prime}}^{N}(\ldots)\right] \delta_{r}^{N}(\ldots) \\
& \times\left[\prod_{f \in \mathcal{J}^{i j}, f \neq r} \delta_{f}^{N}\left(\tilde{h}_{l(f, \tilde{\mathcal{T}})}\right)\right]
\end{aligned}
$$

Each $\delta^{N}$ in the last line integrates with its associated $\tilde{h}_{l(f, \tilde{\mathcal{T}})}$, and we get

$$
\begin{aligned}
A^{\mathcal{G}_{p}} & =\frac{(\lambda \bar{\lambda})^{p}}{\left[\delta^{N}(e)\right]^{p}} \int \prod_{\ell \in \mathcal{L}_{p} \backslash \tilde{\mathcal{T}}} \mathrm{d} h_{\ell}\left[\prod_{f^{\prime} \in \mathcal{F}^{i j} \cup \mathcal{F}^{\widehat{i j}}} \delta_{f^{\prime}}^{N}(\ldots)\right] \delta_{r}^{N}(\ldots) \\
& \leq \frac{(\lambda \bar{\lambda})^{p}}{\left[\delta^{N}(e)\right]^{p}}\left[\delta^{N}(e)\right]^{\left|\mathcal{F}^{i j}\right|+\left|\mathcal{F}^{\widehat{i j}}\right|+1} .
\end{aligned}
$$

One can use any of the three jackets of the graph to derive a bound. Using the jacket which yields the best estimate, we always have

$$
A^{\mathcal{G}_{p}} \leq \frac{(\lambda \bar{\lambda})^{p}}{\left[\delta^{N}(e)\right]^{p}}\left[\delta^{N}(e)\right]^{\mid \frac{\mathcal{F} \mid}{3}+1}
$$


and by Eq. (7) we have

$$
2 p-4 p+|\mathcal{F}|-|\mathcal{P}|=-\sum_{b \in \mathcal{P}} g_{b} \Rightarrow|\mathcal{F}|=2 p+\sum_{b \in \mathcal{P}}\left(1-g_{b}\right)
$$

Note that $\sum_{b \in \mathcal{P}}\left(1-g_{b}\right) \leq 4$ (and equal 4 if and only if the Core Graph is dual to a manifold). The Core Graph bound ensures that more and more complicated topologies (i.e. topologies which cannot be represented by a Core Graph with $p$ vertices or less) are suppressed at least as $\left[\delta^{N}(e)\right]^{\frac{7-p}{3}}$ in Eq. (29).

\subsection{The $1 / N$ Expansion}

We are now in the position to perform the $1 / N$ expansion of the colored GFT model. In order to evaluate all contributions to the order $\left[\delta^{N}(e)\right]^{-\alpha}$, one lists all (equivalence classes of) Core Graphs up to order $p=3 \alpha+7$. This is a finite problem, hence solvable. Then one computes the amplitude of each Core Graph (which can of course turn out to be much smaller than the value predicted by the Core Graph bound). The free energy is

$$
F(\lambda, \bar{\lambda})=\sum_{p=1}^{3 \alpha+7} \sum_{\left[\mathcal{G}_{p}\right] \in \mathfrak{G}_{p}} C^{\left[\mathcal{G}_{p}\right]}(\lambda, \bar{\lambda}) A^{\left[\mathcal{G}_{p}\right]}+O\left(\left[\delta^{N}(e)\right]^{-\alpha}\right) .
$$

The Core Graphs up to $p=3$ are the graphs $\mathcal{G}_{1}, \mathcal{G}_{2}, \mathcal{G}_{3, a}, \mathcal{G}_{3, b}, \mathcal{G}_{3, c}$ and $\mathcal{G}_{3, d}$ from Fig. 2. The Core Graphs $\mathcal{G}_{1}, \mathcal{G}_{2}, \mathcal{G}_{3, a}$ and $\mathcal{G}_{3, b}$ are dual to the three sphere $S^{3}$. The Core Graphs $\mathcal{G}_{3, a}$ and $\mathcal{G}_{3, b}$ are in the same equivalence class at order 3. The Core Graphs $\mathcal{G}_{3, c}$ and $\mathcal{G}_{3, d}$ are dual to pseudo manifolds: $\mathcal{G}_{3, c}$ has two non-planar bubbles each of genus 1 , while $\mathcal{G}_{3, d}$ has only one non-planar bubble of genus 1. The Core Graph bound ensures that

$$
\begin{gathered}
A^{\left[\mathcal{G}_{1}\right]} \leq\left[\delta^{N}(e)\right]^{2}, \quad A^{\left[\mathcal{G}_{2}\right]} \leq\left[\delta^{N}(e)\right]^{\frac{5}{3}}, \quad A^{\left[\mathcal{G}_{3, a}\right]} \leq\left[\delta^{N}(e)\right]^{\frac{4}{3}}, \\
A^{\left[\mathcal{G}_{3, c}\right]} \leq\left[\delta^{N}(e)\right]^{\frac{2}{3}}, \quad A^{\left[\mathcal{G}_{3, d}\right]} \leq\left[\delta^{N}(e)\right] .
\end{gathered}
$$

Contributions coming from Core Graphs at higher order are at most of order $\delta^{N}(e)$. Direct computation shows that

$$
\begin{aligned}
& A^{\left[\mathcal{G}_{1}\right]}=\left[\delta^{N}(e)\right]^{2}, \quad A^{\left[\mathcal{G}_{2}\right]}=\left[\delta^{N}(e)\right], \quad A^{\left[\mathcal{G}_{3, a}\right]}=\left[\delta^{N}(e)\right]^{0}, \\
& A^{\left[\mathcal{G}_{3, c}\right]}=\frac{1}{\delta^{N}(e)} \int \mathrm{d} h \mathrm{~d} u \delta^{N}\left(h u^{-1} h^{-1} u\right), \quad A^{\left[\mathcal{G}_{3, d}\right]}=\left[\delta^{N}(e)\right]^{0} .
\end{aligned}
$$

and $A^{\left[\mathcal{G}_{3, c}\right]}=1$ if $G=U(1)$, or $A^{\left[\mathcal{G}_{3, c}\right]}=\left[\delta^{N}(e)\right]^{-1} \sum_{j} 1 \approx\left[\delta^{N}(e)\right]^{-2 / 3}$ for $G=S U(2)$. The partition function of the colored Boulatov model develops as

$$
F(\lambda, \bar{\lambda})=C^{\left[\mathcal{G}_{1}\right]}(\lambda, \bar{\lambda})\left[\delta^{N}(e)\right]^{2}+O\left(\left[\delta^{N}(e)\right]\right),
$$

and all graphs contributing to the dominant order are dual to manifolds homeomorphic with the three sphere $S^{3}$. 


\section{Conclusion}

Throughout this paper, ${ }^{7}$ when evaluating amplitudes, we repeatedly replaced $\delta^{N}(g h) \delta^{N}(h)$ by $\delta^{N}(g) \delta^{N}(h)$. Of course, the two expressions are equal only in the $N \rightarrow \infty$ limit. For finite $N$, the equality holds only in the power counting sense, i.e. up to corrections which vanish in the $N \rightarrow \infty$ limit. All the "=" signs in this paper should be interpreted in this sense (including in our final expansion (39)). On the contrary, the " $\leq$ " signs (i.e. the bounds on the graph amplitudes) hold at finite $N$, and all "=" signs can be traded for " $\leq$ ". Indeed, both the face routing and the behavior of the amplitude under 1-Dipole contractions hold as upper bounds by performing only changes of variables and bounding, at the last step, some $\delta^{N}(g)$ by $\delta^{N}(e)$ which holds at finite $N$.

The colors of CGFT are crucial in establishing the $1 / N$ topological expansion encoded in Eq. (29) and theorem 1. The definitions of bubbles, Dipoles, jackets, connectivity graphs routing trees and Core Graphs are given in terms of colors. A legitimate question is which of these concepts can be extended for non-colored GFT models. Whereas bubbles can be defined for more general models [25], they generically exhibit singularities. Dipole contractions cannot be defined in general (in the absence of colors, one does not know how to reconnect the half lines). The jackets are ill-defined for generic models (the same face can contain two strands of the same line: deleting such a face does not lead to a ribbon graph). Connectivity and Core Graphs cannot be defined in generic models. As almost all tools required to establish the $1 / N$ expansion of CGFT do not generalize to non-colored models, it is highly unlikely that the latter admit a $1 / N$ expansion. ${ }^{8}$

Using the $1 / N$ expansion performed in this paper, one can now investigate the generalization in higher dimensions of many features of matrix models. One can study for instance the dominant spherical sector, explore a double scaling limit, investigate a possible phase transition and its relation to conformal field theory, generalize the KPZ relations, and the list goes on.

\section{Acknowledgements}

Research at Perimeter Institute is supported by the Government of Canada through Industry Canada and by the Province of Ontario through the Ministry of Research and Innovation.

\section{References}

[1] 't Hooft, G.: A planar diagram theory for strong interactions. Nucl. Phys. B 72, 461 (1974).

[2] Gross, D.J., Miljkovic, N.: A nonperturbative solution of $D=1$ string theory. Phys. Lett. B 238, 217 (1990)

\footnotetext{
7 We thank an anonymous referee for recalling us this subtlety.

8 The conspicuous lack of results in this direction also points to the same conclusion.
} 
[3] Gross, D.J., Klebanov, I.R.: One-dimensional string theory on a circle. Nucl. Phys. B 344, 475-498 (1990)

[4] Di Francesco, P., Ginsparg, P.H., Zinn-Justin, J. : 2-D gravity and random matrices. Phys. Rept. 254, 1-133 (1995). [hep-th/9306153]

[5] David, F.: A model of random surfaces with nontrivial critical behavior. Nucl. Phys. B 257, 543 (1985)

[6] Kazakov, V.A., Migdal, A.A., Kostov, I.K.: Critical properties of randomly triangulated planar random surfaces. Phys. Lett. B 157, 295-300 (1985)

[7] Boulatov, D.V., Kazakov, V.A., Kostov, I.K., et al. : Analytical and numerical study of the model of dynamically triangulated random surfaces. Nucl. Phys. B 275, 641 (1986)

[8] Kazakov, V., Kostov, I.K., Kutasov, D.: A Matrix model for the two-dimensional black hole. Nucl. Phys. B 622, 141-188 (2002). [hep-th/0101011]

[9] Brezin, E., Itzykson, C., Parisi, G., Zuber, J.B.: Planar diagrams. Commun. Math. Phys. 59, 35 (1978)

[10] Gross, M.: Tensor models and simplicial quantum gravity in $>2-\mathrm{D}$. Nucl. Phys. Proc. Suppl. 25, 144 (1992)

[11] Ambjorn, J., Durhuus, B., Jonsson, T.: Three-dimensional simplicial quantum gravity and generalized matrix models. Mod. Phys. Lett. A 6, 1133 (1991)

[12] Sasakura, N.: Tensor model for gravity and orientability of manifold. Mod. Phys. Lett. A 6, 2613 (1991)

[13] Freidel, L. : Group field theory: an overview. Int. J. Theor. Phys. 44, 1769 (2005). [arXiv:hep-th/0505016]

[14] Oriti, D.: The Group Field Theory Approach to Quantum Gravity: Some Recent Results. [arXiv:0912.2441 [hep-th]]

[15] Boulatov, D.V.: A model of three-dimensional lattice gravity. Mod. Phys. Lett. A 7, 1629 (1992). [arXiv:hep-th/9202074]

[16] Freidel, L., Louapre, D.: Ponzano-Regge model revisited. I: Gauge fixing, observables and interacting spinning particles. Class. Quant. Grav. 21, 5685 (2004). [arXiv:hep-th/0401076]

[17] Baratin, A., Oriti, D.: Group Field Theory with Non-Commutative Metric Variables. [arXiv:1002.4723 [hep-th]]

[18] Engle, J., Pereira, R., Rovelli, C.: Flipped spinfoam vertex and loop gravity. Nucl. Phys. B 798, 251 (2008). [arXiv:0708.1236 [gr-qc]]

[19] Livine, E.R., Speziale, S.: A new spinfoam vertex for quantum gravity. Phys. Rev. D 76, 084028 (2007). [arXiv:0705.0674 [gr-qc]]

[20] Freidel, L., Krasnov, K.: A new spin foam model for 4D gravity. Class. Quant. Grav. 25, 125018 (2008). [arXiv:0708.1595 [gr-qc]]

[21] Geloun, J.B., Gurau, R., Rivasseau, V.: EPRL/FK group field theory. Europhys. Lett. 92, 60008 (2010). [arXiv:1008.0354 [hep-th]]

[22] Alexandrov, S., Roche, P.: Critical Overview of Loops and Foams. arXiv:1009.4475 [gr-qc]

[23] Gurau, R.: Colored Group Field Theory. [arXiv:0907.2582 [hep-th]]

[24] Gurau, R.: Topological graph polynomials in colored group field theory. Ann. Henri Poincaré 11, 565 (2010). [arXiv:0911.1945 [hep-th]] 
[25] Gurau, R.: Lost in translation: topological singularities in group field theory. Class. Quant. Grav. 27, 235023 (2010). arXiv:1006.0714 [hep-th]

[26] Freidel, L., Gurau, R., Oriti, D.: Group field theory renormalization-the 3D case: power counting of divergences. Phys. Rev. D 80, 044007 (2009). [arXiv:0905. 3772 [hep-th]]

[27] Magnen, J., Noui, K., Rivasseau, V., Smerlak, M.: Scaling behaviour of threedimensional group field theory. Class. Quant. Grav. 26, 185012 (2009). [arXiv: 0906.5477 [hep-th]]

[28] Geloun, J.B., Magnen, J., Rivasseau, V.: Bosonic Colored Group Field Theory. [arXiv:0911.1719 [hep-th]]

[29] Geloun, J.B., Krajewski, T., Magnen, J., Rivasseau, V.: Linearized group field theory and power counting theorems. Class. Quant. Grav. 27, 155012 (2010). [arXiv:1002.3592 [hep-th]]

[30] Gurau, R., Rivasseau, V.: Parametric representation of noncommutative field theory. Commun. Math. Phys. 272, 811 (2007). [arXiv:math-ph/0606030]

[31] Lins, S.: Gems, Computers and Attractors for 3-Manifolds. Series on Knots and Everything, vol. 5. ISBN: 9810219075/ISBN-13: 9789810219079

[32] Ferri, M., Gagliardi, C.: Crystallisation moves. Pac. J. Math. 100(1) (1982)

[33] Bonzom, V., Smerlak, M.: Bubble divergences from cellular cohomology. Lett. Math. Phys. 93, 295 (2010). [arXiv:1004.5196 [gr-qc]]

[34] Grosse, H., Wulkenhaar, R.: Renormalisation of phi**4 theory on noncommutative $\mathrm{R}^{* *} 4$ in the matrix base. Commun. Math. Phys. 256, 305 (2005). [arXiv:hepth/0401128]

[35] Gurau, R., Magnen, J., Rivasseau, V., Vignes-Tourneret, F.: Renormalization of non-commutative phi**4(4) field theory in $x$ space. Commun. Math. Phys. 267, 515 (2006). [arXiv:hep-th/0512271]

[36] Rivasseau, V., Vignes-Tourneret, F., Wulkenhaar, R.: Renormalization of noncommutative phi**4-theory by multi-scale analysis. Commun. Math. Phys. 262, 565-594 (2006). [hep-th/0501036]

\section{Razvan Gurau}

Perimeter Institute for Theoretical Physics,

31 Caroline St., Waterloo,

Ontario N2L 2Y5, Canada

e-mail: rgurau@perimeterinstitute.ca

Communicated by Carlo Rovelli.

Received: November 30, 2010.

Accepted: March 4, 2011. 